\title{
ASSOCIATION BETWEEN ANEMIA AND BONE PROFILE IN NON-DIALYSIS DEPENDENT CHRONIC KIDNEY DISEASE ANEMIA AND BONE PROFILE IN CHRONIC KIDNEY DISEASE
}

\author{
Shamsa Kanwal, Muhammad Aamir, Khurram Mansoor*, Naveed Asif**, Muhammad Tanveer Sajid* \\ Armed Forces Institute of Pathology/National University of Medical Sciences (NUMS) Rawalpindi Pakistan, *Armed Forces Institute of Urology/National \\ University of Medical Sciences (NUMS) Rawalpindi Pakistan, ${ }^{* *}$ Combined Military Hospital Quetta/National University of Medical Sciences (NUMS) Pakistan
}

\section{ABSTRACT}

Objective: To determine association between iron deficiency anemia and bone profile in cases of chronic kidney disease not undergoing dialysis.

Study Design: Comparative cross sectional study.

Place and Duration of Study: Department of Chemical Pathology and Endocrinology, Armed Forces Institute of Pathology in collaboration with department of Nephrology, Armed Forces Institute of Urology, Rawalpindi, from Sep 2018 to Oct 2019.

Methodology: A total of 300 chronic kidney disease patients who were not dependent on any kind of dialysis were included in our study. Chronic kidney disease was confirmed according to National Kidney Foundation/Kidney Disease Outcome Quality Initiative criteria. Hemoglobin, Mean corpuscular volume and ferritin levels were used to diagnose iron deficiency anemia. Association of Calcium, inorganic Phosphorous, Magnesium, Alkaline phosphatase and Parathyroid Hormone (PTH) was determined with iron deficiency anemia.

Results: Out of 300 patients studied 201 (67\%) were females while 99 (33\%) were males. Mean age of participants was 43.51 \pm 5.652 years. Mean duration of chronic kidney disease was $4.36 \pm 3.712$ years. One hundred and forty one $(47 \%)$ patients had no anemia while 159 (53\%) had presence of iron deficiency anemia. After applying binary logistic regression analysis hypocalcemia, hypophosphatemia and hypomagnesemia were noted to have a statistically significant association with iron deficiency anemia in the target sample size.

Conclusion: This study revealed a high frequency of iron deficiency anemia in non-dialysis chronic kidney disease patients. Hypocalcemia, hypophosphatemia and hypomagnesemia were found having strong association with presence of iron deficiency anemia in non-dialysis dependent chronic kidney disease patients.

Keywords: Anemia, Bone profile, Chronic kidney disease.

This is an Open Access article distributed under the terms of the Creative Commons Attribution License (https://creativecommons.org/licenses/by-nc/4.0/), which permits unrestricted use, distribution, and reproduction in any medium, provided the original work is properly cited.

\section{INTRODUCTION}

Chronic kidney disease (CKD), being a multisystem disorder, has been challenging worldwide. The effects of this illness don't remain confined to the renal system but affect the overall homeostasis of the human body $^{1}$. High prevalence of CKD has been noted in recent years in both, the developed and developing countries of the world ${ }^{2,3}$. Management options for this chronic debilitating condition include various modalities ranging from use of medications to renal transplant ${ }^{4}$.

Progression of CKD is associated with a number of complications, which include high incidence of hyperlipidemia, cardiovascular disease, anemia and metabolic bone disease. CKD cases need assessment for presence of these complications ${ }^{1}$. Hematological and biochemical abnormalities have been commonly encountered clinical problems among the patients of $\mathrm{CKD}^{5}$. Anemia in CKD can result from iron, folate, or vitamin B12 deficiency, decreased erythropoietin (EPO) levels

Correspondence: Dr Shamsa Kanwal, House No. 12, Street 9, Sector J, DHA Phase II, Islamabad Pakistan

Received: 16 Mar 2020; revised received: 07 Apr 2020; accepted: 28 Apr 2020 and systemic inflammation. Anemia of chronic disease is usually found in CKD but Iron deficiency anemia (IDA) has now been an established entity with CKD may it be dialysis dependent or non-dependent ${ }^{6,7}$. Anemia has specially been studied in non-dialysis dependent CKD patients. These patients require more specialized surveillance for all the hematological and biochemical profile to decrease morbidity and mortality. If timely diagnosis and treatment of IDA is not done, then these patients develop complications like derangement of bone profile earlier than those without anemia. This may worsen CKD, as a result, patient may become dependent on dialysis for survival7,8.

Biochemical profile derangement has also been a focus of study for researchers as well as clinicians 8 . Bone profile has been one of the important parameters which gets deranged in renal disease and may lead to serious consequences if left untreated ${ }^{9}$. Literature is available on relationship of bone profile parameters with anemia and overall mortality of patient. Nalado et $a l^{9}$. concluded that anemia was found in $>50 \%$ of the patients of CKD. Severity of CKD and hypocalcemia emerged as the factors affecting the presence as well as 
severity of anemia in their study. Xiong et al10 in their systematic review and metanalysis published in 2019 involving more than two hundred thousand patients concluded significant association of hypomagnesemia with mortality in patients with CKD and ESRD.

Limited research is available on association of bone profile and other biochemical abnormalities with IDA in CKD patients in our set up, however burden of disease is high globally ${ }^{9}$. If this multisystem illness is not catered for in all the aspects especially its hematological and biochemical manifestations it may lead to serious consequences which may add insult to the injury and a non-dialysis CKD case may become dialysis dependent one. Limited research is available on association of bone profile and other biochemical abnormalities with IDA in CKD patients in our set up. We therefore planned this study with the objective to determine the association between IDA and bone profile in CKD at a tertiary care setup of Rawalpindi.

\section{METHODOLOGY}

This comparative cross sectional study was conducted at department of Chemical Pathology and Endocrinology, Armed Forces Institute of Pathology (AFIP) in collaboration with department of Nephrology and Armed Forces Institute of Urology (AFIU) Rawalpindi from September 2018 to October 2019 after taking approval from Institutional Review Board (IRB), AFIP Rawalpindi (FC-CHP17-6/READ-IRB/18/908). WHO sample size calculator was used for sample size calculation with population prevalence proportion of $14 \%{ }^{7}$. Non probability consecutive sampling technique was used. CKD cases (Stage 3-5) who were not dependent on any type of dialysis between the age of 18 and 65 years were included in the study. Diagnosis of CKD was done according to National Kidney Foundation/ Kidney Disease Outcome Quality Initiative (NKF/ KDOQI) 2002 ${ }^{10-14}$. Estimated GFR was calculated using CKD-EPI equation. Patients who had vitamin B-12, folate deficiency or were on replacement therapy, low EPO levels with or without treatment, had NSAIDs abuse, vitamin D supplementation, any autoimmune disorder, malignancy or anemia prior to diagnosis of CKD were excluded from the study. Patients with other comorbid diseases causing derangement of $\mathrm{Ca}$, $\mathrm{Mg}$, Vitamin D, iPTH or ALP were also excluded from the study. Study was explained and informed consent was taken. About $3 \mathrm{~mL}$ of venous blood sample was collected in K EDTA and gel tube. Hemoglobin $(\mathrm{Hb})$ and Mean corpuscular volume (MCV) were measured using flow cytometer. Serum ferritin and iPTH were measured on IMMULITE® 2000 immunoassay system by using solid phase two-site chemiluminescent enzyme labelled immunometric assay. Serum $\mathrm{Ca}, \mathrm{Mg}$, iPO4, Albumin, ALP levels were measured on ADVIA ${ }^{\circledR} 1800$ Clinical Chemistry random access auto analyzer by using Cresophthalein complexone (CPC method), Xylidyl Blue, Phosphomolybdate UV, Bromocresol green (BCG) and Nitrophenyl phosphate (pNPP) methods respectively. Internal quality control was run before analysis of samples. All parameters were analyzed according to the standard operational principles of laboratory. IDA for both males and females was defined as total hemoglobin $(\mathrm{Hb})<12 \mathrm{~g} / \mathrm{dl}$, mean corpuscular volume (MCV) <80fl and serum ferritin level $\leq 15 \mathrm{ng} /$ $\mathrm{mL}^{12-15}$. Other reference ranges used were; albumin corrected Ca: 2.15-2.57 mmol/L, Mg: 0.6-0.9 mmol/L, iPO4 :0.8-1.62 mmol/L, iPTH: 0.8-6 pmol/L and ALP: 56-365 IU/L ${ }^{16}$.

Chi-square was applied to look for the correlation of $\mathrm{Ca}, \mathrm{iPO} 4, \mathrm{Mg}$, ALP and iPTH levels with the presence of IDA among the target population. Once correlation was established then extent of correlation was determined by the binary logistic regression analysis. All statistical analysis was performed using SPSS-24. The $p$-value of $\leq 0.05$ was considered to be significant.

\section{RESULTS}

Target population was all CKD patients not dependent on dialysis but with the application of inclusion and exclusion criteria and consent of the individuals 300 patients were finally recruited in the study from whom data could be collected and analyzed. Out of 300 non-dialysis dependent CKD patients studied in the given time period $201(67 \%)$ were females while $99(33 \%)$ were males (figure). Mean age of participants

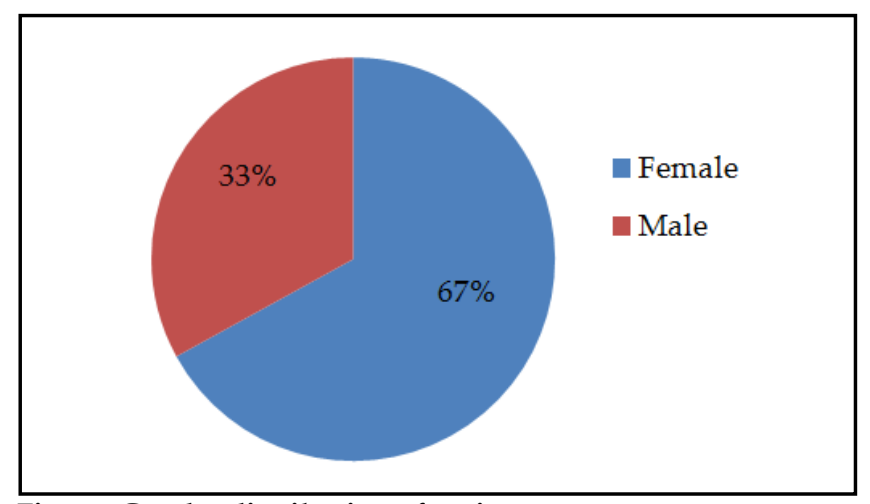

Figure: Gender distribution of patients.

was $43.51 \pm 5.652$ years and mean duration of CKD was $4.36 \pm 3.712$ years. Total $117(39 \%)$ cases were in 
CKD stage 3, 138 (46\%) in stage 4 and $45(15 \%)$ in stage 5 (non-dialysis dependent). Out of 141 (47\%) had no anemia while $159(53 \%)$ had presence of IDA. Table showed parameters of bone profile included in the study. It showed that hypocalcemia, hypophosphatemia and hypomagnesemia had a statistically significant association with existence of IDA in the target population ( $p$-value $<0.05)$ while high ALP and PTH levels had no statistically significant relationship with presence of IDA in this study ( $p$-value $>0.05$ ).

Table: Outcome of various variables studied in the analysis: chi-square test.

\begin{tabular}{|c|c|c|c|}
\hline \multirow[t]{2}{*}{ Factors Studied } & \multicolumn{2}{|c|}{$\begin{array}{c}\text { Iron Deficiency } \\
\text { Anaemia } \\
\end{array}$} & \multirow{2}{*}{$\begin{array}{c}p- \\
\text { value }\end{array}$} \\
\hline & No, n (\%) & Yes, n (\%) & \\
\hline Normocalcemia & $82(58.1)$ & $69(43.4)$ & \multirow{2}{*}{0.011} \\
\hline Hypocalcaemia & $59(41.9)$ & $90(56.6)$ & \\
\hline Hyperphosphatemia & $84(59.5)$ & $55(34.6)$ & \multirow{2}{*}{$<0.001$} \\
\hline Hypophosphatemia & $57(40.5)$ & $104(65.4)$ & \\
\hline Normomagnesemia & $108(76.5)$ & $92(57.8)$ & \multirow{2}{*}{0.593} \\
\hline Hypomagnesemia & $33(23.5)$ & $67(42.2)$ & \\
\hline $\begin{array}{l}\text { Alkaline phosphatase } \\
\text { within reference limits }\end{array}$ & $106(75.2)$ & $119(74.8)$ & \multirow{2}{*}{0.947} \\
\hline $\begin{array}{l}\text { High alkaline } \\
\text { phosphatase }\end{array}$ & $35(24.8)$ & $40(25.2)$ & \\
\hline $\begin{array}{l}\text { Parathyroid hormone } \\
\text { levels within reference } \\
\text { limits }\end{array}$ & $95(67.3)$ & $108(67.9)$ & \multirow[t]{2}{*}{0.919} \\
\hline $\begin{array}{l}\text { High parathyroid } \\
\text { hormone level }\end{array}$ & $46(32.7)$ & $51(32.1)$ & \\
\hline
\end{tabular}

\section{DISCUSSION}

Patient suffering from CKD pass through a lot of phases as the stages of CKD advance. Medical treatment, dialysis and transplant are the modes of treatment which could cater for many aspects of this debilitating illness but still a lot of aspects remain unaddressed and quality of life of the CKD patients remain compromised in one way or another. Park et $\mathrm{al}^{7}$, examined management options of IDA in CKD patients (non-dialysis). They concluded that anemia is one of the major problems among the patients of CKD and various options to treat them have variable effects. They noted parenteral iron preparations have a better outcome as compared to the oral iron therapy7. Our study though did not involve any therapy, but our results showed a high prevalence of IDA among the non-dialysis dependent CKD patients.

Damasiewicz et all1, Discussed in detail all the causes leading to derangement of bone profile and its consequences like increased incidence of fractures among CKD patients. Renal osteodystrophy has multidimensional basis and one of the most important fac- tors involved in pathogenesis has been the derangement of the bone profile secondary to the renal disease.

Ferritin being circulating iron storage protein is increased in proportion to body iron stores. However, ferritin is also an acute phase reactant that can increase independently of iron status. A very low ferritin level $(<15 \mathrm{ng} / \mathrm{mL})$ is diagnostic of iron deficiency, but a higher ferritin level can't be used to eliminate the possibility of iron deficiency in individuals with comorbidities. The sensitivity for iron deficiency increases as the cutoff is raised, but specificity decreases; different values have been suggested for different population ${ }^{12}$.

Sonkar et $a^{16}$, in their study published in 2018 with the objective to look for the association of hyperparathyroidism with anemia in CKD concluded that better control of PTH hypersecretion is required to have a good management of anemia and mineral metabolism. They showed that anemia has a strong association with deranged PTH levels and hypocalcemia. Our results were a bit different, though hypocalcemia emerged as a strong factor to predict IDA but PTH levels had no significant relationship with presence of anemia in our study.

Xiong et al ${ }^{10}$. in their metanalysis concluded that there was a very strong association between hypomagnesemia and the risk of mortality in patients with ESRD (HR 1.32; 95\% CI 1.19-1.47; $p<0.00001$ ) (hypomagnesemia vs. normal magnesium or hypermagnesemia) after multivariable adjusted. On the other hand, hypermagnesemia was inversely associated with mortality in patients with CKD and ESRD (HR 0.86; 95\% CI $0.79-0.94 ; p=0.001$ ) (per unit increase). They also observed a significant association between hypermagnesemia and decreased risk of cardiovascular mortality (HR 0.71; 95\% CI 0.53-0.97, $p=0.03$ ) in the adjusted model. Moreover, subgroup analysis noted that hypomagnesemia was strongly associated with increased allcause mortality in hemodialysis patients (HR 1.29; 95\% CI 1.12-1.50; $p=0.0005$ ) (hypomagnesemia vs. normal magnesium or hypermagnesemia) ${ }^{10}$. Our study only revolved around one component of cardiovascular parameter and that is anemia and hypomagnesemia emerged as a strong predictor for IDA and supported the conclusions given by Xiong et al.

Hypophosphatemia was also significantly associated with IDA in non-dialysis dependent CKD patients in our study. Studies of Hofman et al17, and Wolf et al18, with different study designs on anemia and CKD concluded that Iron and phosphate metabolism has a complex relationship with each other. Usually 
hyperphosphatemia occurs in CKD patients. Hyperphosphatemia was noted in our non-anemic CKD cases. However, a different finding observed in our study is that most of our patients with IDA had hypophosphatemia as compared to those without anemia. More research is needed in this regard to ascertain this association.

Sumida et $a l^{19}$, in their recent study concluded that ALP levels predict the overall mortality in patients suffering from CKD. Deranged ALP levels were significantly associated with decreased hemoglobin levels. Our results did not support their results as ALP levels were not found correlating with existence of anemia in our study population.

Imtiaz et $a^{20}$, conducted a review of the epidemiology of CKD in Pakistan in year 2018 and found a prevalence of $29.9 \%(24.2-35.1 \%)$ in men and $32.5 \%$ $(24.8-41.3 \%)$ in women ${ }^{20}$. We also noted a high percentage $(67 \%)$ of CKD in women in our study. Risk of developing anemia is double in CKD as compared to that in general population. Anemia in CKD is associated with disturbance of sleep cycle, cognitive impairment, progression of CKD stage, cardiovascular comorbidities, and higher mortality ${ }^{20}$.

The study results signify that CKD patients with IDA are at a high risk of a lot of biochemical abnormalities and risk of hypocalcemia and hypomagnesemia increases in presence of IDA. Therefore, patients who have anemia should be investigated frequently for biochemical abnormalities as compared to those without iron deficiency anemia.

Selection of samples from the patients of one center is a major limitation of our study. It should have been a population-based study recruiting patients from multiple centers. We only included cases with IDA. We didn't correlate Vitamin D in our study. Studies in future with large sample size, sophisticated design including all types of anemias in CKD may generate results that are more generalizable.

\section{CONCLUSION}

High frequency of IDA among non-dialysis dependent CKD patients. ALP and PTH levels did not show any association with presence of IDA but hypocalcemia, hypophosphatemia and hypomagnesemia were found having a strong link with presence of IDA in non-dialysis dependent CKD patients.

\section{CONFLICT OF INTEREST}

This study has no conflict of interest to be declared by any author.

\section{REFERENCES}

1. Fraser SD, Blakeman T. Chronic kidney disease: identification and management in primary care. Pragmat Obs Res 2016; 7(1): 21-32.

2. O'Callaghan-Gordo C, Shivashankar R, Anand S, Ghosh S, Glase J, Gupta R, et al. Prevalence of and risk factors for chronic kidney disease of unknown etiology in India: secondary data analysis of three population-based cross-sectional studies [published correction appears in BMJ Open 2019; 9(3): e023353-56.

3. Ruwanpathirana T, Senanayake S, Gunawardana N, Munasinghe A, Ginige S, Gamage D, et al. Prevalence and risk factors for impaired kidney function in the district of Anuradhapura, Sri Lanka: a cross-sectional population-representative survey in those at risk of chronic kidney disease of unknown aetiology. BMC Public Health 2019; 19(1): 763-67.

4. Grill AK, Brimble S. Approach to the detection and management of chronic kidney disease: What primary care providers need to know. Can Fam Physician 2018; 64(10): 728-35.

5. Aoun M, Karam R, Sleilaty G, Antoun L, Ammar W. Iron deficiency across chronic kidney disease stages: Is there a reverse gender pattern? Barretti P, ed. PLoS One 2018; 13(1): e0191541-45.

6. Kato H, Nangaku M, Hirakata H, Wada T, Hayashi T, Sato H, et al. Rationale and design of observational clinical research in chronic kidney disease patients with renal anemia: renal prognosis in patients with hyporesponsive anemia to erythropoiesisstimulating agents, darbepoeti $\mathrm{N}$ alfa (BRIGHTEN Trial). Clin Exp Nephrol 2018; 22(1): 78-84.

7. Park H, Liu X, Henry L, Harman J, Ross EA. Trends in anemia care in non-dialysis-dependent chronic kidney disease (CKD) patients in the United States (2006-2015). BMC Nephrol 2018; 19(1): 318-22.

8. Ko GJ, Obi Y, Tortorici AR, Kalantar-Zadeh K. Dietary protein intake and chronic kidney disease. Curr Opin Clin Nutr Metab Care 2017; 20(1): 77-85.

9. Nalado AM, Mahlangu JN, Waziri B, Durate R, Paget G, Olurunfemi $G$, et al. Ethnic prevalence of anemia and predictors of anemia among chronic kidney disease patients at a tertiary hospital in Johannesburg, South Africa. Int J Nephrol Renovasc Dis 2019; 12(3): 19-32.

10. Xiong J, He T, Wang M, Nie L, Zhang Y, Wang Y, et al. Serum magnesium, mortality, and cardiovascular disease in chronic kidney disease and end-stage renal disease patients: a systematic review and meta-analysis. J Nephrol 2019; 13(2): 1-22.

11. Damasiewicz MJ, Nickolas TL. Rethinking Bone Disease in Kidney Disease. JBMR Plus 2018; 2(6): 309-22.

12. Tran TN, Eubanks SK, Schaffer KJ, Zhou CY, Linder MC. Secretion of ferritin by rat hepatoma cells and its regulation by inflammatory cytokines and iron. Blood 1997; 90(2): 4979-82.

13. Ali T, Idrees MK, Shoukat, Akhtar SF. Left ventricular hypertrophy among predialysis chronic kidney disease patients: Sindh institute of urology and transplantation experience. Saudi J Kidney Dis Transpl 2017; 28(4): 1375-80.

14. Keith DS, Nichols GA, Gullion CM, Brown JB, Smith DH. Longitudinal follow-up and outcomes among a population with chronic kidney disease in a large managed care organization. Arch Intern Med 2004; 164(6): 659-63.

15. Chapter 1: Diagnosis and evaluation of anemia in CKD. Kidney Int Suppl (2011), 2012; 2(4): 288-91.

16. Sonkar SK, Singh HP, Sonkar GK, Pandey S. Association of vitamin $\mathrm{d}$ and secondary hyperparathyroidism with anemia in diabetic kidney disease. J Family Med Prim Care 2018; 7(4): 815-18.

17. Hofman JMG, Eisenga MF, Diepenbroek A, Nolte IM, Dam BV, Westerhuis R, et al. Switching iron sucrose to ferric carboxymal- 
tose associates to better control of iron status in hemodialysis patients. BMC Nephrol 2018; 19(1): 242-45.

18. Wolf M, Chertow GM, Macdougall IC, Kaper R, Krop J, Strauss W. Randomized trial of intravenous iron-induced hypophosphatemia. JCI Insight 2018; 3(23): e124486.

19. Sumida K, Molnar MZ, Potukuchi PK, Thomas F, Lu JL, Obi Y, et al. Prognostic significance of pre-end-stage renal disease serum alkaline phosphatase for post-end-stage renal disease mortality in late-stage chronic kidney disease patients transitioning to dialysis. Nephrol Dial Transplant 2018; 33(2): 264-73.

20. Imtiaz S, Salman B, Qureshi R, Drohlia MF, Ahmad A. A review of the epidemiology of chronic kidney disease in Pakistan: A global and regional perspective. Saudi J Kidney Dis Transpl 2018; 29(1): 1441-51. 